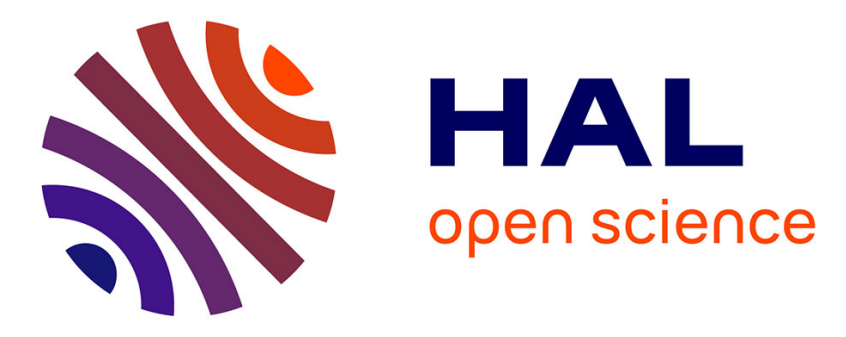

\title{
Hardening mechanisms at grain boundaries: a microscopic approach
}

\author{
A. George
}

\section{To cite this version:}

A. George. Hardening mechanisms at grain boundaries: a microscopic approach. Revue de Physique Appliquée, 1988, 23 (4), pp.479-490. 10.1051/rphysap:01988002304047900 . jpa-00245795

\section{HAL Id: jpa-00245795 https://hal.science/jpa-00245795}

Submitted on 1 Jan 1988

HAL is a multi-disciplinary open access archive for the deposit and dissemination of scientific research documents, whether they are published or not. The documents may come from teaching and research institutions in France or abroad, or from public or private research centers.
L'archive ouverte pluridisciplinaire HAL, est destinée au dépôt et à la diffusion de documents scientifiques de niveau recherche, publiés ou non, émanant des établissements d'enseignement et de recherche français ou étrangers, des laboratoires publics ou privés. 


\title{
Hardening mechanisms at grain boundaries: a microscopic approach
}

\author{
A. George \\ Laboratoire de Physique du Solide, UA CNRS 155, Ecole des Mines, Parc de Saurupt, \\ 54042 Nancy Cedex, France
}

(Reçu le 26 mai 1987, accepté le 3 juillet 1987)

\begin{abstract}
Résumé. - Les mécanismes de durcissement par les joints de grains sont illustrés à 1'aide d'observations récentes dans des bicristaux de silicium. Après quelques rappels élémentaires sur la structure des joints et des dislocations intergranulaires, appliqués au joint de coíncidence $\Sigma=9$, les processus de dissociation des dislocations dans les joints de grain et de transmission des dislocations d'un grain à 1 'autre sont discutés. A température modérée, ces mécanismes opèrent difficilement et n'empêchent pas la formation au voisinage des joints de régions beaucoup plus écrouies que l'intérieur des grains et où 1 'on observe, dès la limite élastique, des réseaux de barrières de LomerCottrell, de nombreux indices de glissement dévié et des concentrations de contraintes très élevées, éventuellement opposées à la contrainte appliquée. La généralisation de ces observations à d'autres types de joints et d'autres matériaux est discutée brièvement.

Abstract. - Grain-boundary induced hardening mechanisms are illustrated by recent observations in silicon bicrystals. Basic features of grain boundary structure and grain boundary dislocations are given in the case of the $\Sigma=9$ twin boundary. The dissociation of dislocations in grain boundaries and the possibility of direct dislocation transmission from one crystal to the other across the interface are discussed. At moderate temperatures, such mechanisms are difficult and not able to prevent the formation of work hardened regions in the neighbourhood of grain boundaries, right at the onset of plastic deformation. Those regions contain networks with Lomer-Cottrell locks and show evidence of profuse cross-slip. Large stress concentrations arise, that may be against the applied stress. The relevance of these findings for other kinds of grain boundaries and other materials is briefly discussed.
\end{abstract}

\section{Introduction}

Grain boundaries play an important part in the strength of materials. Yet, although extensive work hás been done on single crystals as well as on grain boundaries, predicting the mechanical properties of a polycrystalline aggregate from his knowledge on single crystal plasticity is still a challenge to the materials scientist.

There is little doubt that a most important problem in polycrystals is the need for maintaining the cohesion between grains when deformation proceeds. As these compatibility problems are assumed to be thoroughly dealt with by $C$. Rey in this conference, the emphasis here is put on microscopic aspects of the problem, namely interactions and reactions of dislocations with grain boundaries.

Since grain boundaries introduce some discontinuity in both the geometry of slip and elastic properties, interaction with the dislocations which carry slip within the grains is unavoidable. There are various ways in which grain boundaries can modify dislocation dynamics and dislocation distributions : Because of the crystallographic discontinuity, grain boundaries are primarily assumed to hinder disloca- tion motion and to act as obstacles. A first set of questions then arises : How strong obstacles are grain boundaries? Are they able to sustain long pile ups ? Or can some transmission of dislocations take place across them, from one grain to the other? If such a possibility of direct transmission exists, what level of stress is required for it operates and what are the rules for the choice of the slip system(s) that could be activated in the other grain by a given dislocation incoming at the grain boundary ? But grain boundaries are not necessarily obstacles to dislocation motion, they have also been postulated to be efficient dislocation sinks i.e. they could absorb dislocations, preventing then pile up formation. The capacity of grain boundaries for dislocation absorption has often been founded on the intuitive view that grain boundaries have less ordered structures than crystals and that such poorly ordered structures can easily accomodate the topological disorder that is present in the core of a 'islocation. In fact, grain boundaries have ordered structures i.e. solid interfaces are built with specific structural units between which dislocations or defects can be defined. The same topological rule applies as in crystals : a dislocation line can- 
not end at a grain boundary unless it is connected to other dislocations, with the total Burgers vector kept constant at nodes or for any reaction.

Therefore the absorption of a dislocation by a grain boundary occurs via a dissociation into grain boundary dislocations with smaller Burgers vectors and when a dislocation is transmitted through a grain boundary either its Burgers vector is common to both adjacent crystals or, in the general case, a grain boundary dislocation has to be created in the interface.

This remark suggests that the efficiency of both dislocation transmission and absorption mechanisms should be rather 1 imited because of the increasing defect content of grain boundaries that would result.

Also questionable, with that view that grain boundaries have ordered structures, is the proposal of several authors that grain boundaries could be among the first dislocation sources at the onset of plastic deformation.

Besides these grain boundary mechanisms and, especially if it is recognized that they cannot prevent or relax pile up stresses, one has to consider intra-grain mechanisms. As shown below, the arrangement of dislocations in the regions adjacent to grain boundaries may be totally different from those in the grain interior and this could be of prime importance for the hardening problem.

None of the above ideas is really new. They have been expressed and discussed in reviews by Hirth [1], Gleiter et al. [2], Smith [3], Priester [4] or Lim and Raj [5], for example. Another exhaustive review is not necessary and this paper simply aims at illustrating the mechanisms listed above with new experimental results obtained in oriented bicrystals of silicon.

As for single crystal work, elemental semiconductors can be viewed as model materials, with the same slip geometry as fcc metals, of very high purity and crystalline perfection, giving the possibility to tune the dislocation velocity by an appropriate choice of testing temperature and, particularly, to freeze dislocation configurations by cooling under load. In addition nearly perfect bicrystals can be grown with wel1-defined grain boundaries and such materials are very well suited to observations by several imaging techniques : X-Ray topography, conventional and High Resolution electron microscopy.

Most results were obtained in the so-called $\Sigma=9$ bicrysta1, whose structural characteristics are given in the next section, as an example introducing to the crystallography of grain boundaries and grain boundary dislocations.

Hardening mechanisms at grain boundaries is a wide topic and the reader is warned that important problems have not received in this overwiew point the attention they would have deserved.

(i) All reported results refer to the first stages of plastic deformation starting from dislocationfree materia1. A11 deformation experiments were performed at temperatures between $0.6 \mathrm{Tm}-0.7 \mathrm{Tm}$ ( $\mathrm{Tm}$ absolute temperature of melting), i.e. $1000 \mathrm{~K}$ $\sim 1150 \mathrm{~K}$ in $\mathrm{Si}$, which corresponds to cold deformation of usual metals : deformation is carried by dislocation glide. Cross-slip is possible, but climb in the grain interior is negligible as well as grain boundary sliding or grain boundary migration. These "high temperature" phenomena are not considered.

(ii) Grain boundaries were supposed to be perfectly clean, so that interactions with dislocations are not complicated by segregation or precipitation phenomena [6].

(iii) Bicrystals are an oversimplification of poly- crystals for many purposes and do not allow us to deal with flow stress -grain size relationships or the difficult problem of determining the strain distribution in polycrystalline aggregates.

2. $\Sigma=9$ boundaries in the diamond cubic structure : An example of ordered interface

The $\Sigma=9$ boundary is a high-angle, symmetric, tilt, coincidence boundary, corresponding to a disorientation of $38.94^{\circ}$ around the [011] axis. The boundary plane is indexed $(1 \overline{2} 2)$ I in lattice I and $(12 \overline{2})_{I I}$ in lattice II. (Indices are transposed from one cubic basis to the other by the rotation matrix). This boundary may be referred to as a second order twin boundary.

The atomic structure of the (12 2$) \quad \Sigma=9$ boundary in the diamond cubic structure can be described as a periodic arrangement of $L$ and $L^{\prime}$ structural units made up of five and seven atom rings. $L$ and $L^{\prime}$ units correspond to each other in a glide $\left(1 / 4[41 \overline{1}]_{I}\right)$ mirror $\left((1 \overline{2} 2)_{I}\right)$ symmetry. According to this model, depicted in Figure 1, the structure has no dangling bonds and each $L$ or $L^{\prime}$ unit can be viewed as the core of a perfect Lomer dislocation [7-11].

In the classical picture of a grain boundary as arrays of dislocations these Lomer dislocations would be labelled as intrinsic, primary dislocations.

If the two lattices I and II were allowed to interpenetrate each other, some lattice points would appear to belong simultaneously to both, forming the coincidence site lattice, CSL, represented in bold 1 ines in figure 1 . It is a $\overline{1 s o}$ convenient for our purpose to introduce the so-called DSC lattice represented in dotted lines in figure 1. Any DSC vector is a linear integer combination of vectors of lattices I and II. Grain boundary dislocations usually have Burgers vectors that are vectors of the DSC lattice.

$\Sigma=9$ boundaries in Si bicrystals were really perfect, since they have been grown from seeds containing a naturally occured twin, but let us assume that, in real bicrystals, some departure from the exact coincidence orientation exists, this should be accounted for by an array of secondary dislocations with DSC Burgers vectors, superimposed to the structure depicted above. As a part of the equilibrium structure of the real boundary, these dislocations would also be regarded as intrinsic.

On the contrary, if a lattice dislocation enters the grain boundary it will le regarded -or the reaction products it forms with existing dislocations of the intrinsic arrays- as extrinsic.

There is no need here for a rigorous treatment of these crystallographic features (see $[12,13]$ ) but a last important feature of grain boundary dislocations has to be mentioned : these dislocations are usually associated with steps in the boundary plane. This is because the boundary plane has a preferred path in the CSL unit cell. Since grain boundary dislocations correspond to shear vectors, which are usually not CSL vectors, steps must be introduced in order to preserve the equilibrium structure of the interface on each side of the dislocations $[14$, 15]. Steps introduce extra energy that has to be taken into account in reactions between grain boundary dislocations. They may impede the mobility of grain boundary dislocations. On the other hand, they play an important role in grain boundary sliding.

Topological requirements alone leave various pos-sibilities to define the step associated to a given DSC vector. However, actual steps, which can be observed by High Resolution Electron Microscopy (HREr: carry useful information on the way the DSC disloca- 
tions have been formed.

The special features of [011] tilt bicrystals should be emphasized as far as plastic deformation is concerned : (i) one Burgers vector 1/2 [011] is common to each crystal and (ii) four (111) slip planes -two in each grain-meet along the [011] direction in the boundary plane.

\section{Remarks on deformation conditions}

The reader will find details on experimental techniques in the original papers. Two points concerning deformation conditions are noteworthy. (i) In all kinds of experiments reported below, except for in situ straining experiments in the high voltage electron microscope (HVEM), the stress was applied in a direction parallel to the boundary plane and close to $\langle 123\rangle$. Therefore, each crystal, considered alone, would have deformed by single slip and, assuming that each grain of the bicrystal deformed homogeneously on this primary slip system, no plastic strain incompatibility would have arised at the grain boundary. Such bicrystals are said to be plas.-

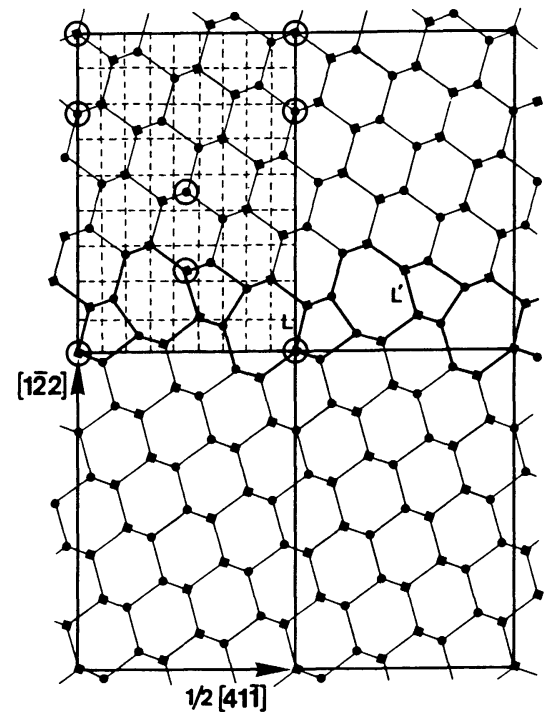

Fig. 1 - $[011]$ projection of the $(1 \overline{2} 2) \quad \sum=9$ symmetrical tilt boundary in the diamond cubic structure - CSL unit cell; --- DSCL unit cell; $\mathbf{0}$, atoms in the plane of the paper ; 9 , atoms $1 / 4[011]$ above.

tically compatible. They could also be defined as isoaxial. (This is not to deny the importance of incompatibilities but simply because, even in this fully compatible situation, dislocation configurations in and near grain boundaries are really involved and difficult to analyse. A microscopic investigation in $\Sigma=9$ bicrystals deformed in non-compatible conditions, for example with a stress axis inclined on the boundary plane might be a next step of this work).

(ii) The applied shear stress, $\tau$, was variable depending on the kind of experiment considered. In HVEM in situ straining experiments it was estimated from the observed dislocation velocity. $\tau$ will be indicated in figure headings.

4. Grain boundaries as dislocation sinks. Dissociation into arain boundary dislocations

A first evidence that dislocations can dissociate when meeting a $\Sigma=9$ boundary is given by Figure 2 .
This thin foil had been used for in situ straining experiments in a HVEM operated at $400 \mathrm{kV}$, cooled down and the dark-field micrograph presented was taken later with a JEOL 200 CX microscope (X. Baillin, A. Jacques and J. Pelissier, unpublished results). In the area presented, some deformation took place only in the grain which is imaged. A group of dislocations piled-up against the boundary plane and this gave rise to grain boundary dislocations which movec in the grain boundary plane away from the line of impact. All dislocations visible in the boundary have the same contrast and their Burgers vector was determined to be the unit DSC vector, parallel to $(1 \overline{2} 2)_{I}$. Those dislocations can move in the grain boundary by pure glide. They are viewed as produced by the dissociation of the leading dislocations of

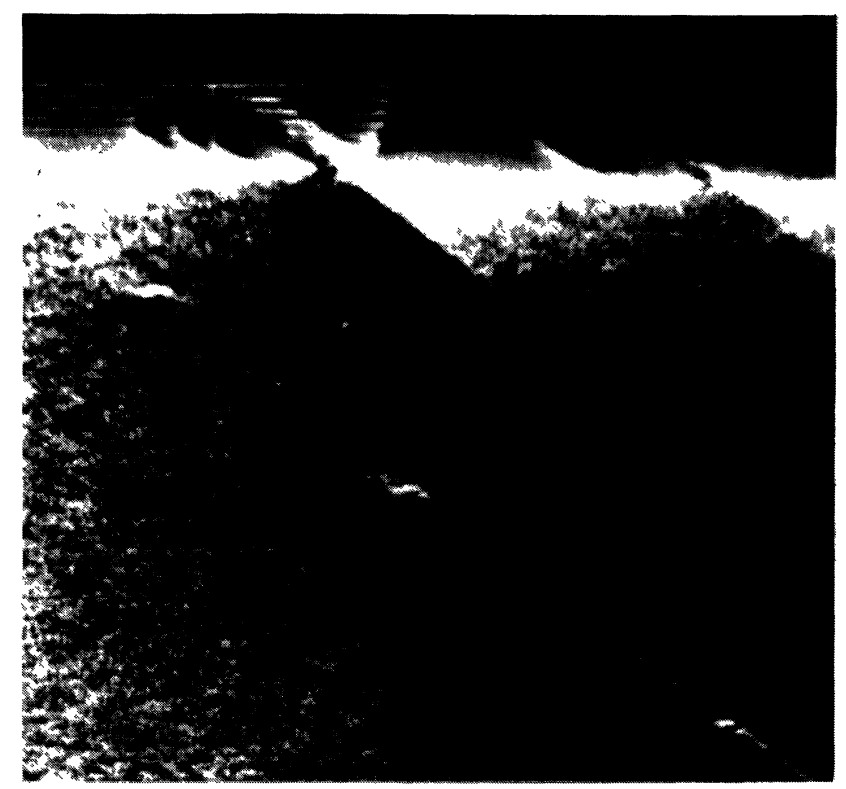

Fig.2 - Conventional TEM observation of a foil that has been prior strained in the HVEM ( $\sim 973 \mathrm{~K}$, $\tau \sim 150 \mathrm{MPa})$. Grain boundary dislocations with Burgers vector $\vec{b}_{g}=1 / 18[41 \overline{1}]$ I glide away from the impact point of primary slip dislocations. (A. Jacques, unpublished results;.

the pile up. Other product dislocations, whose Burgers vectors should have a component normal to $(1 \overline{2} 2 ;$ I and are presumably less mobile since their motion would require some climb, could not be resolved.

A more detailed description of the dissociation process was obtained by high resolution electron microscopic (HREM) observations of bicrystals [16, 17] slightly deformed at $1123 \mathrm{~K}$.

Let us consider in crystal I a group of dislocations of the primary slip system (Burgers vector, $\mathrm{b}^{\mathrm{I}}: 1 / 2[\overline{1} 10]_{\mathrm{I}}$, slip plane $(11 \overline{1})_{\mathrm{I}}$ ) gliding towards the boundary plane. As they arrive near the interface, dislocations align themselves parallel to it, i.e. along [011]. Primary slip dislocations then become $60^{\circ}$ dislocations $\left(60^{\circ}\right.$ is the angle between $\vec{b}$ and the dislocation line, $\vec{l}$ ), whose core structure is well known, since such orientation is a preferred one for isolated dislocations in the diamond cubic structure. HREM observations have shown that such dislocations are dissociated in their slip, plane in two Shockley partial dislocations $\left(b=\frac{1}{6}\right.$ $<112>$ ), with an intrinsic stacking fault in between. In present experiments, the dissociation width was found to be quite the same as in the bulk even for the leading dislocations of a pile up, right at the 
grain boundary. One Shockley partial is of the $90^{\circ}$ type $(\vec{b}-\vec{l})$ and the other of the $30^{\circ}$ type. Depending on the sign of the applied stress, the leading dislocation, which touches the grain boundary first, can be of either type.

In figure $3 \mathrm{a}$, the $90^{\circ}$ partial was the leading one and had just touched the boundary. Figure $3 \mathrm{~b}$ shows what is supposed to be the next step : the $90^{\circ}$ partial has dissociated according to :

$$
\begin{aligned}
& \vec{b}_{90^{\circ}} \rightarrow \vec{b}_{c}+\vec{b}_{g} \\
& \text { with } \vec{b}_{c}=\frac{1}{9}[1 \overline{2} 2]_{I}=d \overrightarrow{s c}_{2} \\
& \overrightarrow{\mathrm{b}}_{\mathrm{g}}=\frac{1}{18}[41 \overline{\mathrm{i}}]_{\mathrm{I}}=\mathrm{d} \overrightarrow{\mathrm{sc}}_{1}
\end{aligned}
$$

$\mathrm{dsc} \overrightarrow{\mathrm{c}}_{1}, \mathrm{dsc}_{2}$ are basic vectors of the DSC lattice.

This reaction was achieved by the glide of the dislocation $\vec{b}_{g}$ in the interface, away from the impact point, to which the dislocation $\vec{b}_{c}$ remained attached.

It appears that the trailing $30^{\circ}$ partial was then able to enter the grain boundary, forming at the impact point a residual dislocation of Burgers vector :

$$
\vec{b}_{r}=\vec{b}_{c}+\vec{b}_{30^{\circ}}
$$

which in turn was observed to dissociate into these two components, which implies that at least one of them was mobile, a process which involved climb i.e. non conservative motion- in the grain boundary.

\section{Some remarks may be made}

(i) It is generally assumed that the driving force for dissociation into grain boundary dislocations is a reduction of elastic energies. If the simple $\Sigma \mathrm{b}^{2}$ criterion is used, the first step observed here does not reduce the elastic energy since the two products have normal Burgers vectors.

This suggests that grain boundary dislocations have different and smaller stress fields than crystal dislocations. At the author's knowledge, this problem is not solved. Recently Lim [18] proposed that a grain boundary dislocation of Burgers vector $b$ can be treated as a crystal dislocation but with a reduced Burgers vectors $\mathrm{fb}$ where

$$
f=1+0.21 \log \alpha
$$

$\alpha$ is the ratio of the elastic constants of the "grain boundary phase" to that of the bulk crystal. (The grain boundary is approximated as a thin second phase of a few lattice constants thick having elastic constants that could be the same as exhibited by amorphous materials).

The second step of the absorption process i.e. the absorption of the trailing $30^{\circ}$ partial is facilitated by the emission of the $\vec{b}_{g}$ dislocation which decreases the repulsive force exerted on it. The absorption is further facilitated if the stress field of the $\vec{b}_{c}$ dislocation is smaller than that of a crystal dislocation of same Burgers vector, following the argument of Lim.

(ii) Except for a few particular cases, some of the product dislocations have a Burgers vector with a component normal to the boundary plane. As stated by Pond and Smith [19], the absorption process involves the separation of the product grain boundary dislocations at a rate limited in general by climb or by their interaction with any pre-existing dislocation networks if more general boundaries are considered. Since climb is involved, the efficiency of dislocation absorption by grain boundaries is expected to increase with temperature, together with the diffusion rate. J. Thibault-Desseaux is present1y investigating $\Sigma=9$ bicrystals of silicon that have been deformed at $1473 \mathrm{~K}$ in order to check this point.

From HREM micrographs, atomic steps associated with grain boundary dislocations and the core structure of the latter could be directly determined. A $\vec{b}_{c}$ dislocation is associated to equal and opposite steps in the two grains $i . e$. the average grain boundary plane is not sheared and, if this is taken as the reference, the step height, $\overrightarrow{\mathrm{h}}_{\mathrm{c}}=0$.

other dislocations have non-zero associated steps i.e. they shear the grain boundary plane and their motion would induce some amount of grain boundary
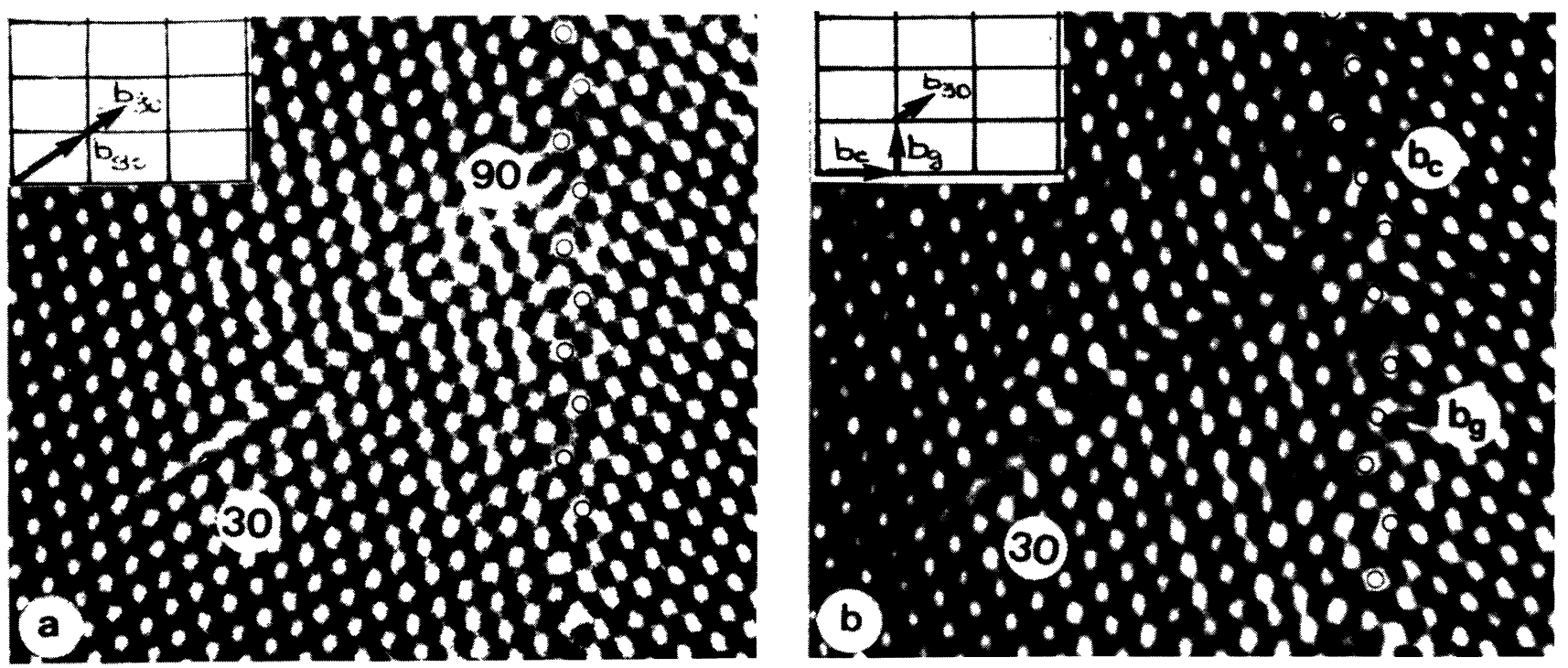

Fig. 3 - (a) A $60^{\circ}$ dislocation arriving at a $\sum=9$ boundary in silicon. In (b;, the leading Shockley partial dislocation, with $90^{\circ}$ character, has dissociated into grain boundary dislocations, $\vec{b}_{c}$ and $\vec{b}_{g}$. The $\vec{b}_{g}$ dislocation has glided in the boundary plane. HREM observations (black atoms) by El Kajbaji and Thibault-Desseaux $[16,17]$. In the attached sketches, the Burgers vectors are indicated in the DSC lattice. 

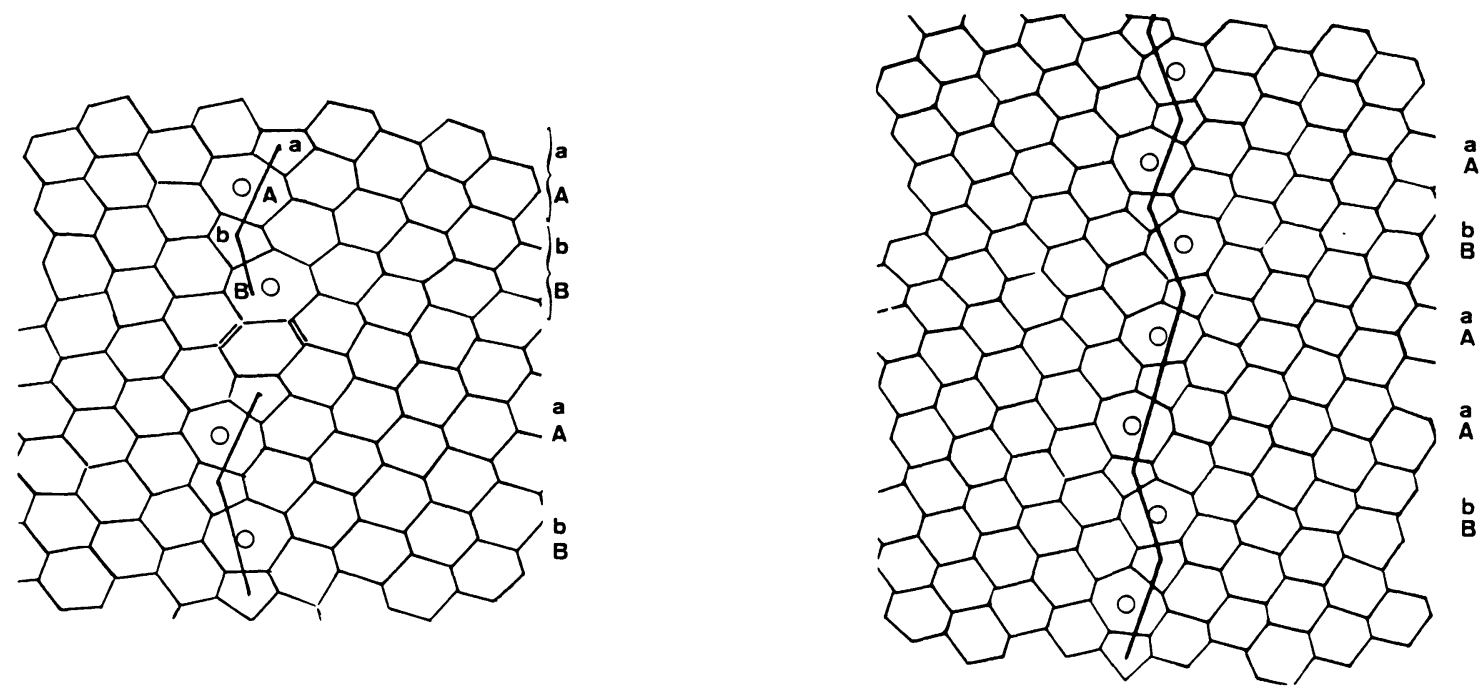

Fig. 4 - Core structures of grain boundary dislocations determined from HREM observations by E1 Kajbaji and Thibault-Desseaux [16]. (a) Dislocation with the Burgers vector $\vec{b}_{\mathrm{C}}=1 / 9[1 \overline{2} 2]_{\mathrm{I}}$. No step is introduced in the boundary. The additional boat-shaped 6 atom ring is a structural unit of the usual (111; $\Sigma=3$ twin boundary. (b) Dislocation with the Burgers vectors $\vec{b}_{\mathrm{g}}=1 / 18[41 \overline{1}]_{\mathrm{I}}$. The boundary plane is sheared by $\overrightarrow{\mathrm{h}}_{\mathrm{g}}=1 / 9\left[1 \overline{\mathrm{L}}^{2}\right]_{\mathrm{I}}$.

migration :

$$
\begin{aligned}
& \overrightarrow{\mathrm{h}}_{\mathrm{g}}=\mathrm{d} \quad \text { with } \mathrm{d}=1 / 9[1 \overline{2} 2]_{\mathrm{I}} \\
& \overrightarrow{\mathrm{h}}_{3 \mathrm{C}}=\overrightarrow{\mathrm{h}}_{\mathrm{r}}=-1.75 \mathrm{~d}
\end{aligned}
$$

Yodels of the core structure of $\vec{b}_{c}$ and $\vec{b}_{g}$ dislocations are shown in Figure 4. (Although it is somewhat out the scope of this paper, it may be noticec. that such cores are well localized and can be reconstructed without any dangling bonds).

other grain boundary dislocations were found. They could be described as the result of reactions between $\vec{b}_{c}, \vec{b}_{g}$ and $\vec{b}_{30}$ dislocations coming from crystal dislocations of crystals I and II [16]. These various configurations suggest that, in the present case, unit grain boundary dislocations are relatively weakly interacting. Transformation of a set of elementary DSC dislocations into complex configurations with non unit DSC Burgers vectors seems to be easily reversible.

The net result of dislocation absorption by $\Sigma=9$ boundaries in the case of symmetrical deformation is the accumulation of $\vec{b}_{c}$ dislocations in the boundary plane. This is easily understood when the symmetric deformation of the two grains is considered. Let us assume that two $60^{\circ}$ dislocations enter the boundary, the one from crystal I and the gther from crystal II. Their dissociation creates two $\vec{b}_{g}$ dislocations of oppoşite signs, two identical $\vec{b}_{c}$ dislocations and two $\vec{b}_{30}$ and $\vec{b} \frac{I}{3}$ dislocations, whose components of Burgers vectors parallel to the grain boundary cancel, whereas normal components add according to the reaction

$$
\vec{b}_{30}^{I}+\vec{b}_{30}^{I I}=\vec{b}_{c}
$$

The two opposite $\vec{b}_{g}$ dislocations, being mobile, certainly annihilate :

$$
\vec{b}_{g}^{I}+\vec{b}_{g}^{I I}=0
$$

and if $\vec{b}_{c}$ dislocations are able to homogenize their distributions, one is left with a subgrain boundary of the same tilt axis superimposed to the initial
$\Sigma=9$ boundary. In agreement with macroscopic observations $[20]$, the misorientation angle between the two components of the bicrystals evolves continuous-ly with strain, increasing in compression, while it would be decreased by a tensile deformation which is expected to create $-\vec{b}_{c}$ dislocations.

\section{Transmission of slip across grain boundaries}

Clear evidence of dislocation transmission across high-angle grain boundaries is rare $[21,22]$ although many authors claimed they have observed it. Preliminary indications for local slip transmission by grain boundaries was gained from slip trace analysis or etch pits distributions. Hook and Hirth $[23,24]$, for example, demonstrated a very high degree of correlation at grain boundaries between narrow slip bands in Fe-Si bicrystals of various orientations. Figure 5 presents a typical slip line configuration in Si bicrystals deformed up to the upper yield point. In initially dislocation-free $\mathrm{Si}$ crystals obtained by the Czochralski pulling technique, the deformation at this stage is highly inhomogeneous and few s.lip lines are present. Very clearly, the deformation has started in one grain and has been propagated in the second through the grain boundary. Such low magnification observations leave however the possibility that the correlation between slip bands in grains $I$ and II results from the activation in grain II of pre-existing dislocation sources by the long range stresses developed by dislocations of grain I when they built pile ups against the boundary. In such a case, one could not really speak of dislocation transmission across the boundary. In ad-dition neither slip lines nor etch pits allow the Burgers vectors of dislocations to be determined.

Most of the evidence for dislocation transmission based on better resolution imaging techniques, as conventional TEM, that can be found in the 1iterature is unfortunately poor, when not totally misleadding. Observations are of the sort shown in Figure 6 . In this micrograph, the two grains of a $\Sigma=9$ bi- 


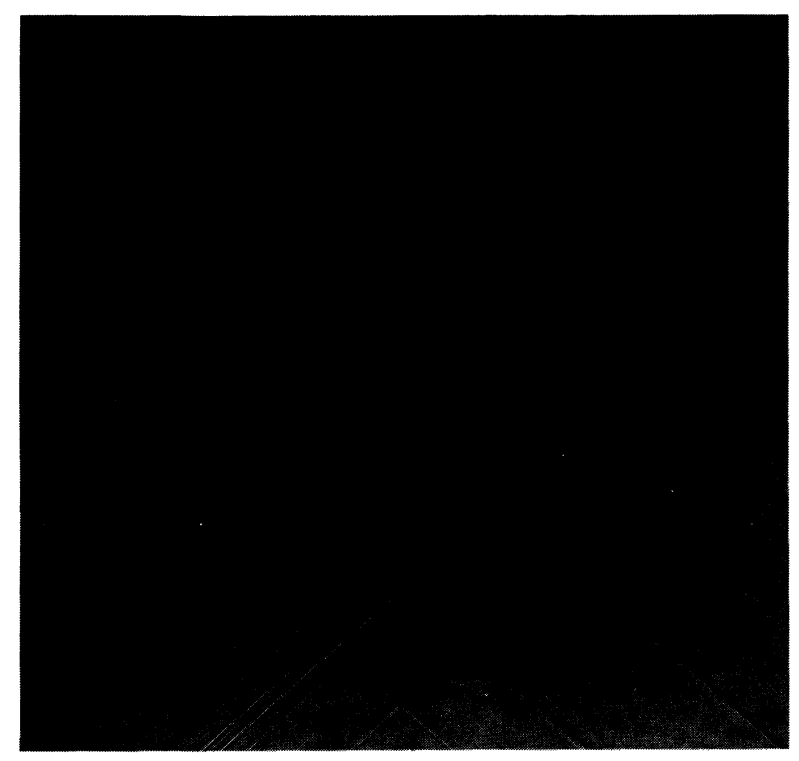

Fig. 5 - S1ip traces in a $\sum=9$ bicrystal of silicon deformed symmetrically in compression at $1023 \mathrm{~K}, \dot{\varepsilon}=8 \times 10^{-6} \mathrm{~s}^{-1}$ up to the upper yield point (nominal stress $\sim 50 \mathrm{MPa}$ ). After [25].

crystal were imaged simultaneously with the common $g=022$. The boundary is made visible on $1 \mathrm{y}$ by dislotions that have been stopped at it. Among these dislocations, one is bulging out from the boundary, which seems to be a good indication that it may have crossed the boundary. In the present case, this dislocation has the $1 / 2$ [011] common Burgers vector and it has probably been transmitted from the other grain (see below). However it could also have been pushed against the boundary and some time later extracted from it backwards as the result of a reversal of the local stress. Experimental indications in favour of such stress reversals will be given in section 6 . Thus such dislocation could have moved forth and back without crossing the boundary.

(Similarly the claimed evidence for the efficiency of grain boundaries as dislocation sources often relies on the simple observation that a higher dislocation density is present in regions adjacent to the boundaries, a fact which obviously is better explained by the barrier that boundaries oppose to dislocations !).

In order to study dislocation transmission, the most convenient experiments are in situ straining experiments. Two kinds of experiments were performed with $\Sigma=9$ silicon bicrystals $[25,26]$ : the one using $\mathrm{X}$-Ray Topography as the imaging technique, with the synchrotron radiation delivered by the LURE-DCI facility to shorten exposure times, and the second using HVEM. In both cases, experiments were interrupted as soon as interesting configurations appeared, the samples cooled down with the load applied and Burgers vector analysis could be done by standard TEM or Xray topography.

In figure 7, a Lang topograph taken after the straining experiments is shown. Dislocations were created from a scratch in the left grain and they accumulate over a large fraction of the grain boundary area. In spite of single slip orientation, the three slip systems with highest Schmid factors, s, could be identified : [ 110$]_{\mathrm{I}}(11 \mathrm{~T})_{\mathrm{I}}(\mathrm{s}: 0.47)$,

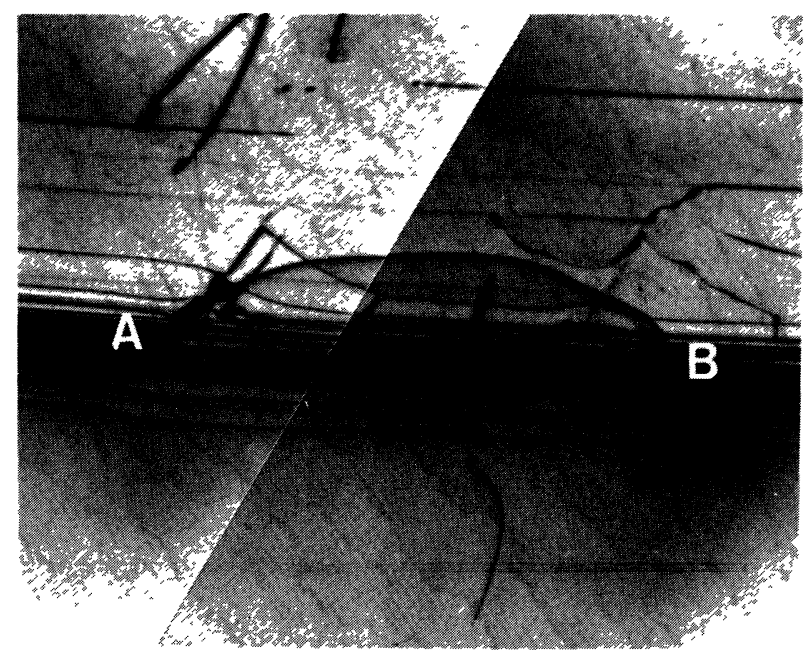

Fig.6 - A possible example of dislocation transmission across a $\sum=9$ grain boundary. $\sum=9$ bicrystal of silicon deformed at $1123 \mathrm{~K}$ slightly, beyond the upper yield point, $\dot{\varepsilon}=8 \times 10^{-6} \mathrm{~s}^{-1}$ shear stress $\sim 8 \mathrm{MPa}$. Load-applied state. The dislocation $A B$ with Burgers vector $1 / 2[011]$ and slip plane (11T) I probably arrived from (111)II in grain II. The glide planes in grains I and II intersect at the boundary along [011]orientation. After [27].

$[011](11 \overline{1})_{\mathrm{I}}(\mathrm{s}: 0.38)$ and $[110]_{\mathrm{I}}(\overline{1} 11)_{\mathrm{I}}(\mathrm{s}: 0.36)$. While most dislocations were stopped, a few groups were able to develop in the second grain, running from the grain boundary towards the grain interior on (1T1'il planes. These groups are not made of primary slip dislocations, but of dislocations having the common $1 / 2$ [011] Burgers vector, ( $s: 0.38)$.

The grain boundary has filtered some of the incoming dislocations.

Qualitatively different is the case depicted in Figure 8. A higher dislocation density was developed in the scratched grain and long range stresses due to dislocation bands are larger, as evidenced by the contrast. At some places at the grain boundary, dislocations belonging to several slip planes were seen to develop in the second grain. Burgers vectors could be determined and the following transmission reactions were suggested :

$$
\begin{aligned}
(1) \vec{b}^{I}=\frac{1}{2}[011]_{I}(11 \overline{1})_{I} \rightarrow \vec{b}^{I I}= & \frac{1}{2}[011]_{I I}(1 \overline{1} 1)_{I I} \\
(2) \vec{b}^{I}=\frac{1}{2}[\overline{1} 10]_{I}(11 \overline{1})_{I} \rightarrow \vec{b}^{I I}= & \frac{1}{2}[\overline{1} 01]_{I I}(1 \overline{1} 1)_{I I}+ \\
& \vec{b}^{G B}=\frac{1}{3}[\overline{1} 2 \overline{2}]_{I} \\
(3) \vec{b}^{I}=\frac{1}{2}[\overline{1} 10]_{I}(11 \overline{1})_{I} \rightarrow \vec{b}^{I I}= & \frac{1}{2}[\overline{1} 10]_{I I}(11 \overline{1})_{I I}+ \\
\text { (4) } \vec{b}^{I}=\frac{1}{2}[110]_{I}(\overline{1} 11)_{I} \rightarrow \vec{b}^{I I}= & \frac{1}{2}[101]_{I I}(\overline{1} 11)_{I I}+ \\
& \vec{b}^{G B}=\frac{1}{9}[1 \overline{2} 2]_{I}
\end{aligned}
$$

(The signs are for dislocations, oriented positively along [011], gliding towards the boundary in crystal $I$ and away from it in crystal II in a deformation in tension).

Because of its 1 imited resolution, X-ray topography cannot prove that transmission of slip results from a one-to-one dislocation process and HVEM experiments were necessary to answer this question. HVEM 
experiments have confirmed that dislocations with the common $1 / 2$ [011] Burgers vector can be transmitted across the boundary not only in symmetrical slip planes (reaction $\mathrm{n}^{\circ} 1$ ) but also on the second (111) plane which contains the bicrystal tilt axis [25]. Reaction $\mathrm{n}^{\circ} 3$ was observed very recently by Jacques, Baillin and Pelissier (unpublished results). This reaction is however difficult and saturates after 2 or 3 dislocations have been transmitted from the head of a given dislocation pile up (Figure 9). Reaction $\mathrm{n}^{\circ} 4$ could not be observed with the orientations used so far for the HVEM samples but is however supported by TEM observations [27]. On the contrary, reaction $n^{\circ} 2$ i.e. direct transmission of primary dislocations in the symmetrical slip system was never observed as expected.

These results can be explained if both crystallographic and dynamic aspects are considered.

From the crystallographic point of view, the direct transmission of a dislocation would require : (i) either a good matching of slip planes of crystals I and II at the boundary or that a small segment of the dislocation may climb or glide on unusual planes [21] in order to surmount the mismatch and catch up the nearest slip plane available in crystal II.

(ii) the creation of a grain boundary dislocation to ensure Burgers vector conservation, since, in general cases, dislocations have not the same Burgers vectors in crystal I and in crystal II.

The first requirement is easily fulfilled in symmetrical bicrystals and is obeyed by all the reactions listed above. The Burgers vector of the grain boundary dislocation that has to be created, $\vec{b} G B$, has been indicated above for any reaction. This' second condition explains why dislocation transmission is so difficult : a supply of extra energy is necessary. A particular case exists in [011] tilt boundaries, since one Burgers vector is common to both grains. In this case, transmission is just crossslip : no defect is created in the boundary and many dislocations can be transmitted from one slip band.
For other reactions, it seems that that the modulus of $\vec{b} G B$ is important to decide which reaction should occur : the smaller $|\vec{b} G B|$, the easier reaction. Here, considering associated steps does not change this conclusion, but this result could not be generalized for other boundaries.

A second very important factor is how efficient the stress concentration created by the pile up of incoming dislocations is to promote the reaction. In our experiments [26], this was estimated as follows. The shear stresses exerted by a crystal I dislocation pile up against the grain boundary were first calculated assuming that the medium was isotropic and the pile up was in equilibrium with an applied shear stress $\tau$.

Since one was interested in a comparison between different slip systems rather than in exact values, the number of dislocations in the pile up was arbitrarily chosen to be 20 and the applied stress taken at $\sim 20 \mathrm{MPa}$. The shear stress exerted in any $\langle 110\rangle$ available slip direction of $\{111\}$ slip planes at any point of crystal II was considered to be the sum of the applied shear stress and of the appropriate component of all pile-up dislocation elastic stress fields.

The relative efficiency of a given pile up in activating the different slip systems in crystal II was displayed by plotting the locus of crystal II points where the shear stress was equal to some arbitrary value. An example is given in Figure 10.

The information contained in the equal shear curve was twofold : (i) the larger the area between the curve and the boundary, the more probable the corresponding slip system activation, (ii) it was to some extent possible to tell from the curve shape whether pile up stresses favoured a direct transmission process or rather the remote activation of nearby pre-existing sources.

This analysis has confirmed the importance of pile up stresses. All the observed transmission reactions are favoured. Direct transmission is favoured for reactions 1,3 and 4 , listed above. On the con-

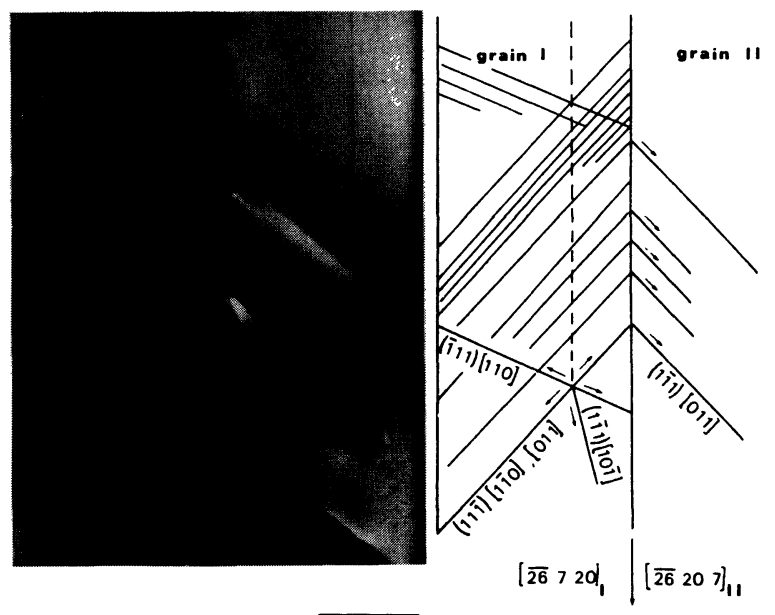

Fig. 7 - Transmission of dislocation groups with $1 / 2$ [011] Burgers vector in a $\sum=9 \mathrm{Si}$ bicrystal deformed for $25 \mathrm{~min}$ at $988 \mathrm{~K}$ and $45 \mathrm{MPa}$. Lang topograph with $\overrightarrow{\mathrm{g}}=1 \overline{1} 3_{\mathrm{I}} / \overline{1} \overline{1} 3_{\mathrm{II}}$ (marker : $1 \mathrm{~mm}$ ) and sketch of identified slip systems. After [25].
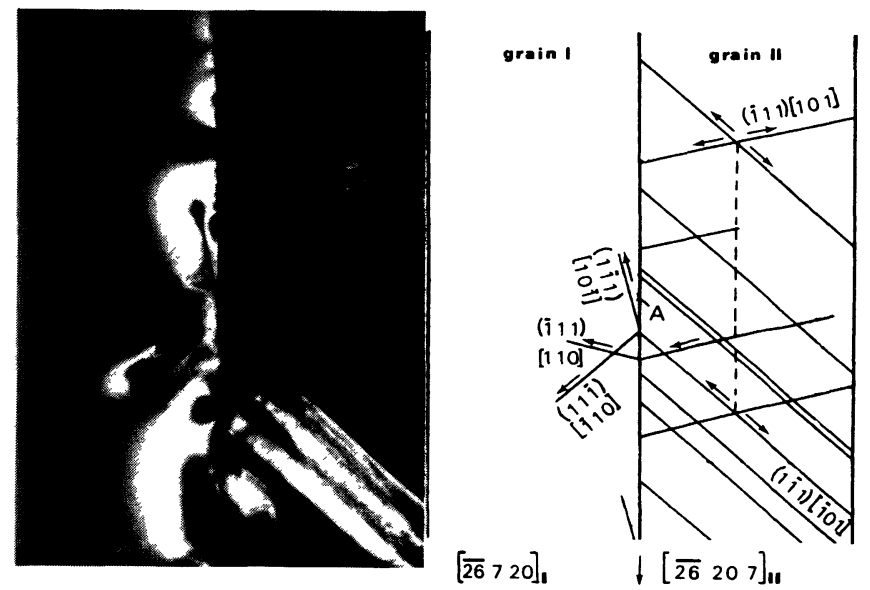

$[\overline{26}>20]$,

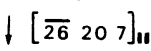

Fig. 8 - Transmission of various types of dislocations by $\sum=9$ grain boundary in a Si bicrystal deformed for $35 \mathrm{~min}$ at $793 \mathrm{~K}$ and $45 \mathrm{MPa}$. Lang topograph with $\overrightarrow{\mathrm{g}}=1 \overline{1} \mathrm{3}_{\mathrm{I}} / \overline{1}^{\mathrm{1}} 3_{\mathrm{II}}$ (marker $: 1 \mathrm{~mm}$ ) and sketch of identified slip systems. After $[25]$. 


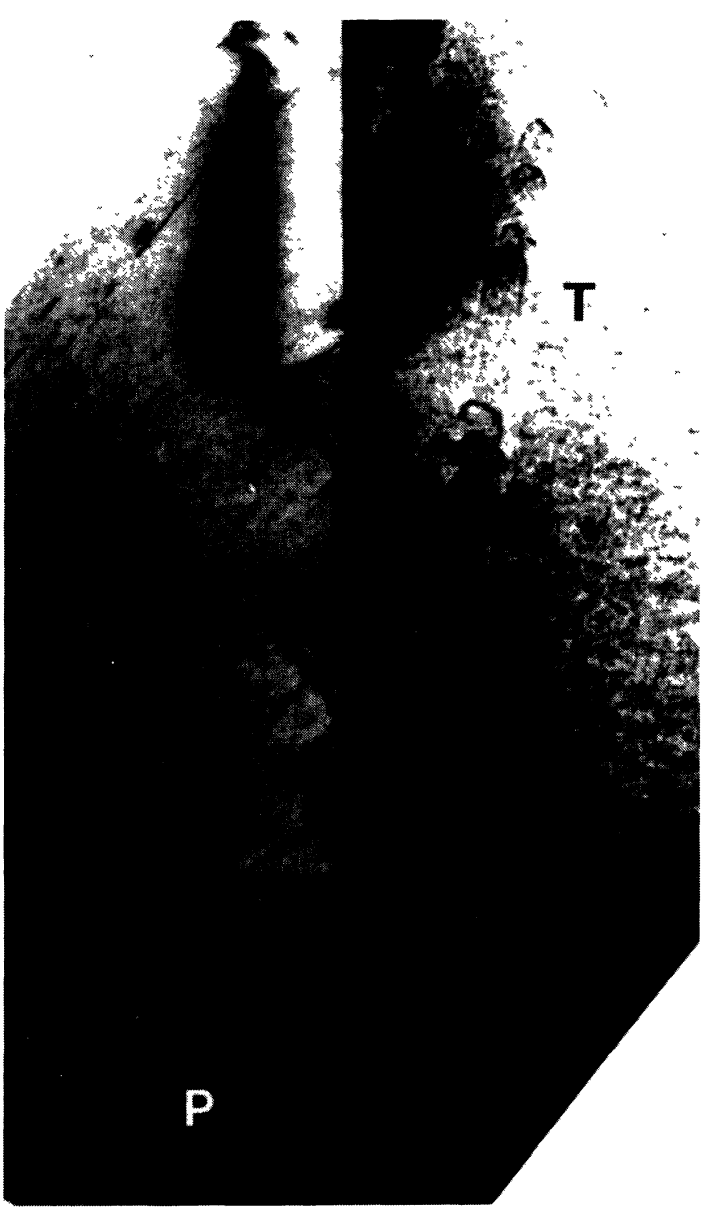

Fig. 9 - Transmission of dislocations T with Burgers vectors $1 / 2[\overline{1} 10]$ II in (111) II planes from pile ups of dislocations $\mathrm{P} 1 / 2[\overline{1} 10]_{\mathrm{I}},(11 \overline{1})_{I}$ (reaction $\mathrm{n}^{\circ} 3$ in the text). TEM micrograph of a foil strained in the HVEM at $\sim 1023 \mathrm{~K}$, $\tau \sim 100 \mathrm{MPa}$ (A.Jacques, X.Baillin, J.Pelissier unpublished work).

trary, reaction 2 may result rather from the activation of pre-existing sources, as observed in HVEM experiments [25].

Remarks :

Since silicon is nearly isotropic, the grain boundary introduces no image forces and no elastic incompatibilities when stressed. In anisotropic crystals very different behaviour can arise and slip transmission may be blocked by repulsive image forces, i.e. dislocations cannot even enter the grain boundary [28].

So far, the mechanism for dislocation transmission across $\sum \neq 9$ boundaries has not been determined. Possible models have been given in $[26,29,20]$ but could not be confirmed, for example by HREM observations. One difficulty is that the dissociation into grain boundary dislocations of the leading partial dislocation does not favour its transmission. In that sense, absorption and transmission can be regarded as antagonist processes.

The main result of our experiments is that dislocation transmission is a very difficult process, which requires large stress concentrations. One major problem appears to be accumulation of residual grain boundary dislocations in the case of localized
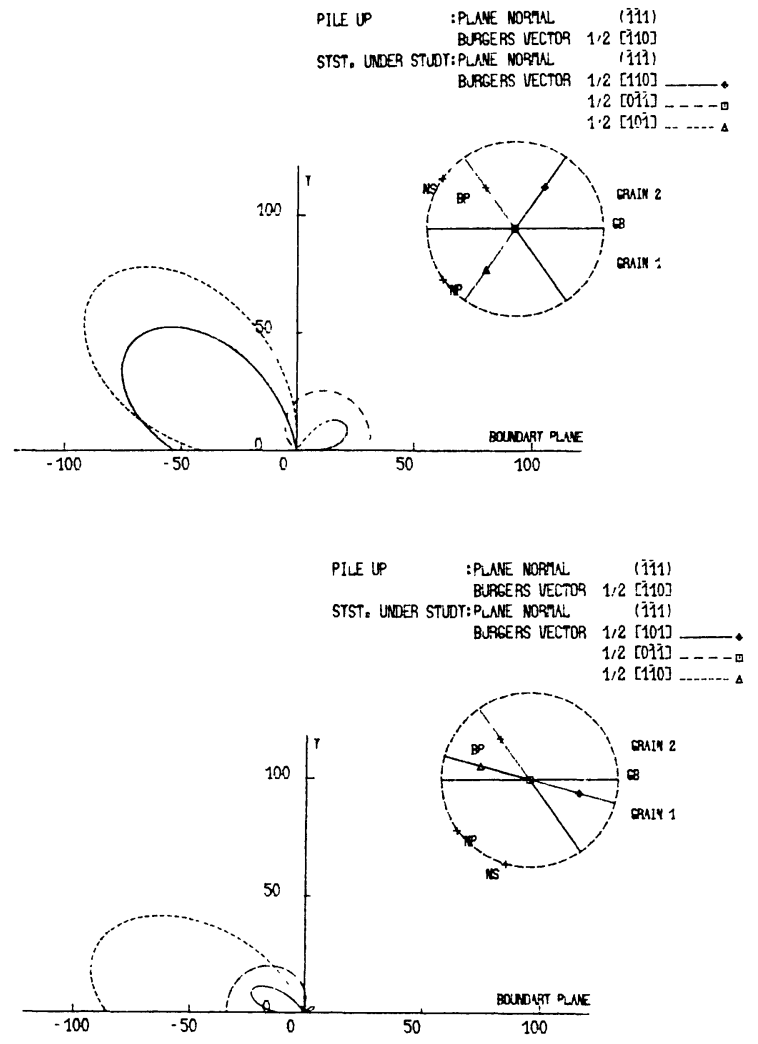

Fig.10 - Examples of equal-shear-stress curves ( $\tau=$ $5 \sigma)$ in slip systems of crystal II resulting from the applied tensile stress $(\sigma=37 \mathrm{MPa})$ and a $20^{\circ}$ dislocation pile up in crystal $\mathrm{I}$. Distances are expressed in units of the lattice parameter. The boundary plane is horizontal. The origin is at the meeting point of the grain boundary and pile up plane, which are viewed end-on, as the slip plane considered in cristal IT.

crossing by several dislocations emitted from the same source. In reactions 2 to 4 above, residual grain boundary dislocations have Burgers vector with components normal to the boundary plane and hence cannot easily move in the grain boundary. Therefore it is probable that the transmission could not proceed any further without introducing cracks at the boundary plane.

6. Dislocation configurations at grain boundaries. lork hardening in regions adjacent to orain boundaries

It is clear from previous sections that, under the "low" temperature conditions considered, grain boundaries are not able to absorb nor to transmit more than a small fraction of incoming dislocations and that they remain a 2-dimensional obstacle for most of them. The blocking of slip by the boundary is expected to create in bicrystals a pile up situation that could not form in a single crystal and this new section reports that dislocations react and rearrange themselves in order to minimize the resulting total stress, forming typical configurations which are never observed in single crystals deformed under similar conditions. 
As pointed out before, for a symmetrical bicrystal subjected to symmetrical loading, plastic compatibility is ensured provided that the deformation proceeds homogeneously in both grains. Our purpose is to demonstrate that even in this highly favourable and simple situation, the inhomogeneity of plastic deformation at the microscopic scale creates quite involved dislocation arrangements near the grain boundary.

In the present experiments, initially dislocationfree bicrystals were strained at an impused constant strain rate. As in single crystals, a stress peak was observed with a maximum defined as the upper yield stress, $\tau_{\text {uy, }}$, and a minimum defined as the 1ower yield stress, $\tau_{\ell y}$.

At the beginning of straining, the stress increases very rapidly since no dislocations are available. Above some stress level, dislocations mutliply in an "avalanche" type process. This sudden increase of the mobile dislocation density allows the imposed strain rate to be achieved under a reduced applied stress, which controls the dislocation velocity. Internal stresses due to dislocation-dislocation interactions also increase with the dislocation density and a minimum flow stress is soon attained.

TEN observations to be reported below were made in bicrystals deformed between the upper and the 10wer yield point, in order to avoid a too high dislocation density near grain boundaries [27]. At this stage of the deformation, it is known that slip is very inhomogeneous, even in single crystals, where several slip directions on several glide planes can be excited, before a single slip situation is established around the lower yield point. In bicrystals, it is thus expected that groups of dislocations may arrive at the boundary from both sides, more or less at random.

Figure 11 presents a typical arrangement of pile ups at the boundary. Long and short pile ups are observed. Long pile ups are often stabilized by secondary dislocations. They consist of several tens of dislocations and may be up to $100 \mu \mathrm{m}$ long. Short pile ups often contain no more than 5 dislocations and are less than $5 \mu \mathrm{m}$ long. It is significant that long pile ups are generally arranged symmetrically on both si- . des of the boundary. Such symmetric pile ups create some stress concentration at the boundary but preserve the symmetry of the bicrystal. On the contrary,

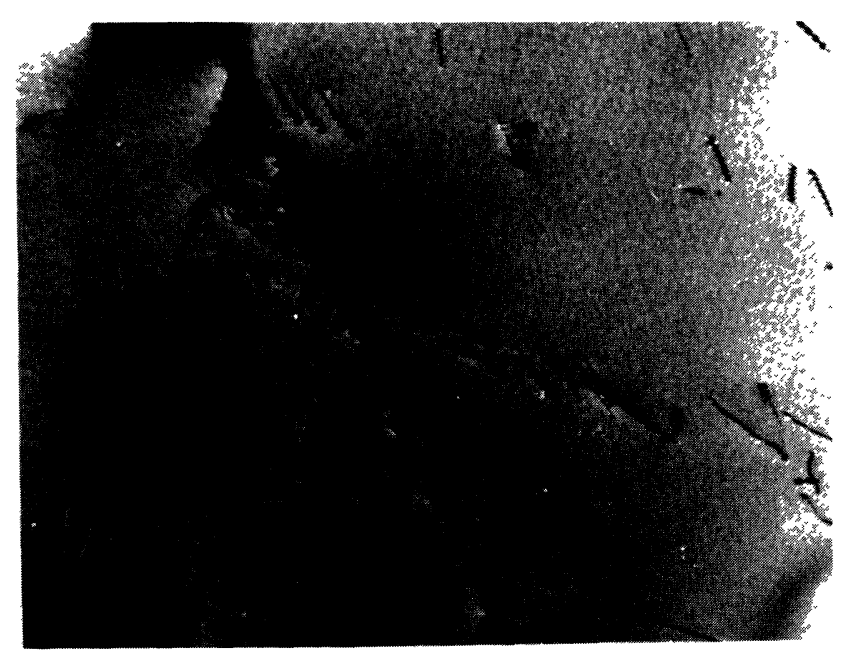

Fig.11 - Typical pile up configuration in a $\sum=9$ Si bicrystal deformed up to the upper yield point at $1023 \mathrm{~K}, \dot{\varepsilon}=8 \times 10^{-6} \mathrm{~s}^{-1}$, nomina 1 strain $8 \times 10^{-3}$, resolved shear'stress $33 \mathrm{MPa}$ (Micrograph M. Martinez-Hernandez).

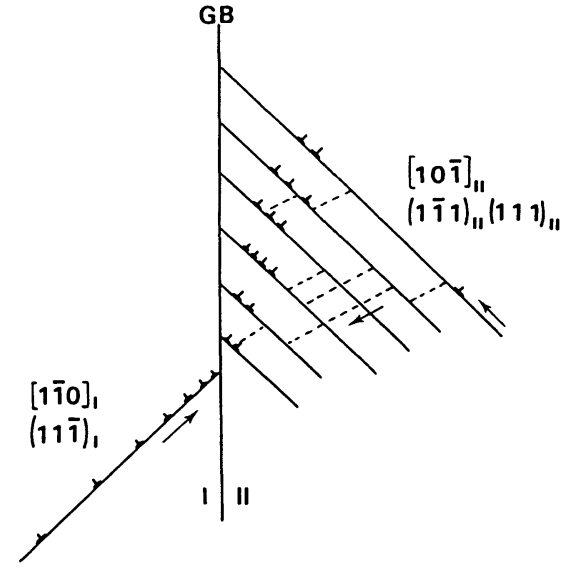

Fig. 12 - Sketch of a configuration in $\sum=9 \mathrm{Si}$ bicrystals deformed as in Fig.11. A long pile up of primary dislocations in grain $I$ is blocked by the boundary. In grain II, primary dislocations arriving on parallel slip planes are blocked before they can reach the boundary by the stress of the pile up in grain I. See $[27]$.

shorter pile ups are staggered every $5 \mu \mathrm{m}$ or so, probably because there is no need to exactly compensate their long range stresses which are much smaller than for longer pile ups.

Figure 12 presents an other possible situation [27] when a long pile up in grain I is not "equilibrated" by a symmetric one in the other grain. Because of the stress barrier caused by the pile up, dislocations that arrived on different parallel slip planes in grain II, could not reach the boundary and form a rough dislocation wall, which has, on average, the character of a subgrain tilt boundary.

Another typical configuration is the dislocation network of figure 13. Because the slip is inhomogeneous, if secondary slip dislocations are activated they can well reach an area of the boundary plane which has not yet been attained by primary slip dislocations. After the upper yield point, mainly primary dislocations multiply and it may be that primaries run into a high density of secondary pile ups at the boundary. The resulting attractive reaction forms Lomer-Cottrell locks [31]. This lead to a network where primary dislocations are stretched out between the Lomer-Cottrell segments, the secondaries, which acted as obstacles, appearing as steps of the ladder structure.

It must be stressed here that secondary dislocations involved in network formation have not been activacted by incompatibility stresses but simply by the applied stress. This is made sure not only by the shape of the network but also through the observation that among all possible reactions of this type, we have observed only those for which the Schmid factor for each reactant was higher than 0.2 .

Such networks are usually observed in stage II of the hardening curve of single crystals. In bicrystals, it can be said that the neighbourhood of the boundary is in an advanced stage of deformation.

Profuse cross-slip is another dislocation process which is mainly involved at an advanced stage of de-ormation in single crystals (transition from stage II to stage III of parabolic hardening) and which occurs near grain boundaries at the yielding of bicrystals. Cross-slip was very frequently observed during in situ straining experiments. It enabled dis locations to escape from the heads of pile ups (Figu- 

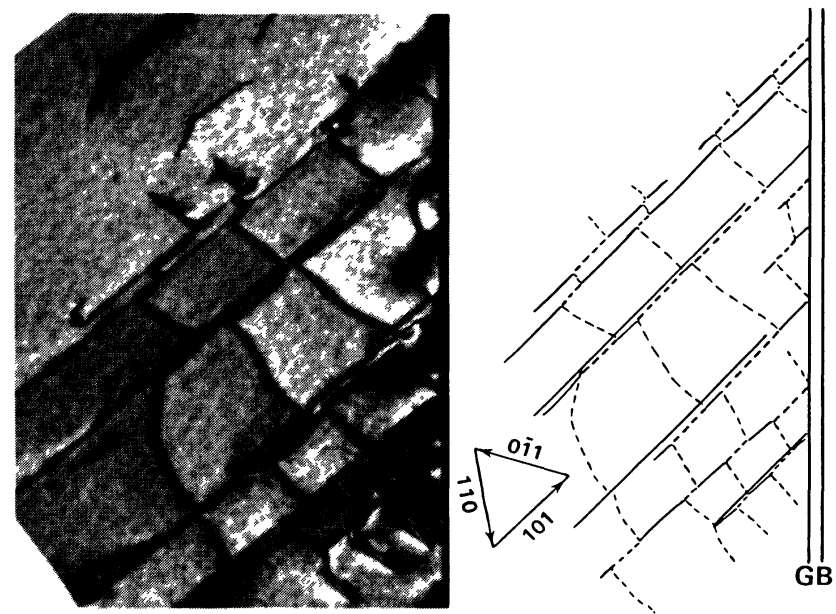

Fig.13 - Dislocation network observed in a $\sum=9 \mathrm{Si}$ bicrystal deformed as in Fig. 11. The curved parts of the dotted lines are secondary dislocations $\left(\vec{b}=1 / 2[01 \bar{I}]_{I},(\overline{1} 11)_{I}\right)$, the full lines are primaries $\left(\vec{b}=1 / 2[1 \overline{1} 0]_{I},(11 \overline{1})_{I}\right)$ and the straight dotted lines are LomerCottrell dislocations $(\vec{b}=1 / 2[10 \overline{1}]$ I $)$. After [27].

re 14) and also provided an efficient way of homoge$\mathrm{n}$ izing the distribution of crystal dislocations trapped in the boundary plane. Baillin et al. [25] reported on the filling of the grain boundary plane with dislocations arriving from a single source by repeated cross-slip. Dislocations first escaped from the pile up that had formed at the boundary, glided over some distance in the cross-slip plane and returned back in a plane parallel to the pile up plane, so that they reached the boundary at a place which was still empty of extrinsic dislocations.

TEM observations in compressed bicrystals have proved that cross-slip near grain boundaries is not only active in thin foils where image forces can help it [32] but also in the bulk. An interesting finding was that cross-slip usually occured symmetrically on both sides of the boundary. This gives fur-
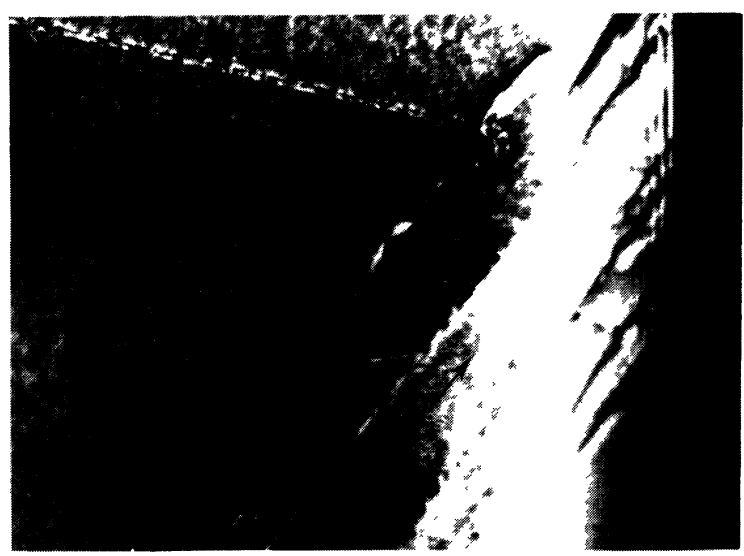

Fig.14 - Dislocations escaping from the heads of pile ups. TEM micrograph of a thin foil strained in the HVEM ( $2923 \mathrm{~K}, \tau \sim 150 \mathrm{MPa}$ ). (A. Jacques et al. unpublished results). The motion of dislocations in the cross-slip plane is made visible by slip traces.

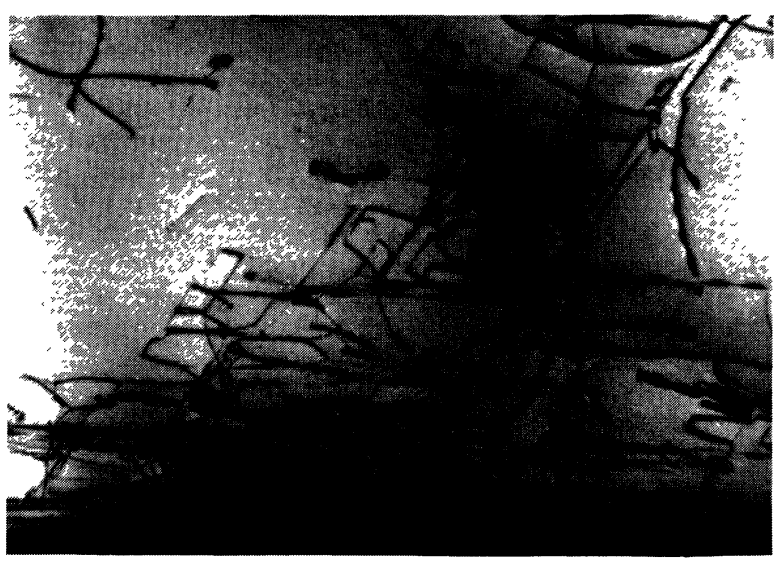

Fig.15 - Prismatic dislocations created by repeated cross-slip. Both the primary slip plane (trace parallel to the grain boundary) and the cross-slip plane are viewed nearly endon. $\Sigma=25$ ( $[001]$ tilt, $\theta=16,26^{\circ}$ ) Si bicrystal deformed at $1023 \mathrm{~K}$, slightly before the lower yield point, nominal strain $7 \times 10^{-3}$, $\tau=11 \mathrm{MPa}$. After [27].

ther indication that dislocation arrangements tend to preserve bicrystals symmetry : since the crossslip reduced the long range stress fields of the original pile ups in grain $I$, the compensating stress fields of the pile ups in grain II had to be reduced in the same way. A a matter of $\mathrm{fact}$, the driving force for cross-slip must have stemmed from internal stresses since the Schmid factor of the applied stress on the cross-slip plane was very low in the orientations that have been investigated. In the configuration of figure 15, complete prismatic loops have been formed by double cross-slip and the formation of such loops where segments screen each others stress fields is a way to minimize long range stresses.

Some of the observations reported above strongly suggest that dislocations arrange themselves in grains I and II so as to compensate mutually their stresses. Such compensation however cannot be perfect in any case and due to dislocation kinetics some degree of overcompensation might occur. If one accepts the idea that stress reversal can occur in a particular region as deformation and time proceed, this implies that backward motion of dislocations must occur.

Such backward motion from the grain boundary was indeed observed during straining experiments in the HVEM (Baillin and Jacques, unpublished work). The configuration shown in figure 16 is supposed to have resulted from a stress reversal during the course of compression at constant strain rate. From the double contrast it is clear that the two dislocations $(a, b)$ have a Burgers vector opposed to that of dislocation (c). Dislocations $(a, b)$ moved from the boundary into the grain interior as indicated by their curvature. In view of the reported difficulties for dislocation transmission across a grain boundary, it is very unlikely that such long dislocations have crossed the boundary. We proposed rather that they were first driven towards the boundary by a -say- positive stress, have aligned parallel to the boundary and moved back into the grain after stress reversal due to dislocation activity in an adjacent area. This newly reversed stress on the other hand has driven dislocation (c) towards the boundary. 
An important point, if this interpretation is correct, is that bow outs of dislocations towards the grain interior cannot be taken as proof that the dislocations have crossed the boundary or were emitted by grain boundary sources.

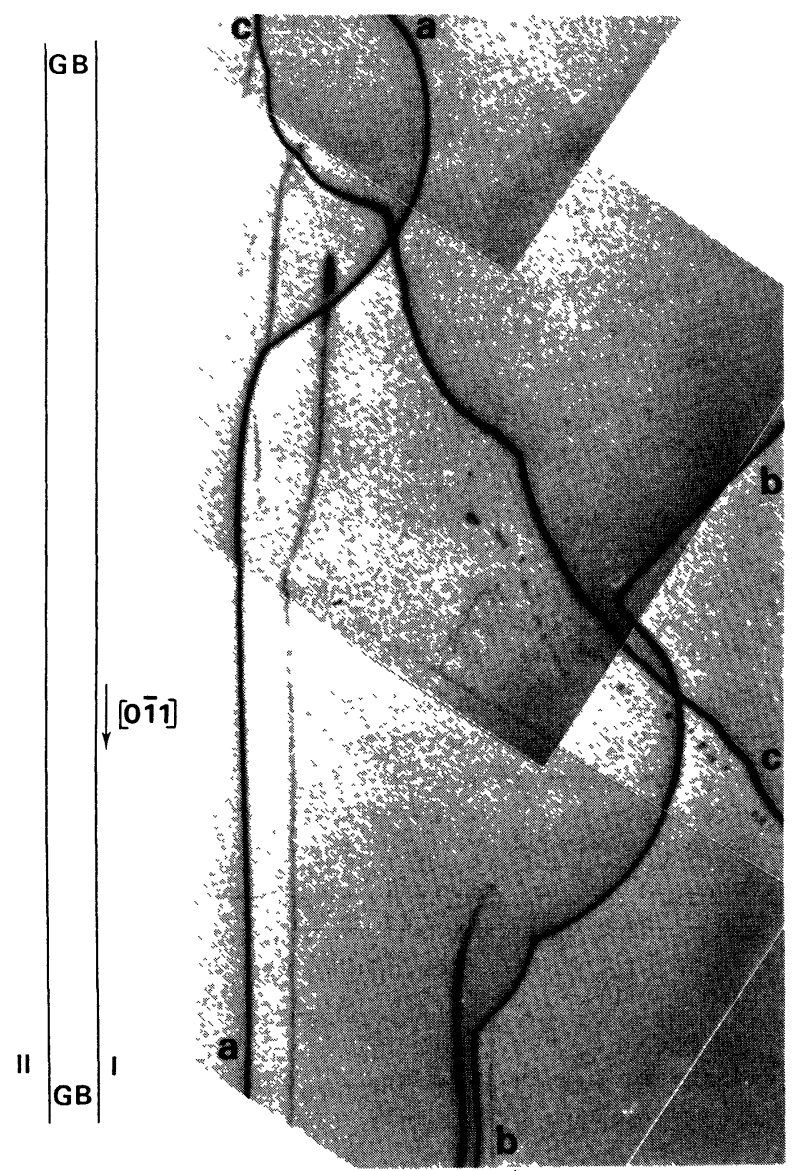

Fig.16 - Dislocations (a) and (b), which had previously been driven towards the boundary, bow out away from it after stress reversal has occured. Dislocation (c), with opposite Burgers vector, is pushed towards the boundary. $\sum=25$ Si bicrystal deformed as in Fig. 15. After $[27]$.

\section{Summary and concluding remarks}

$\Sigma=9$ bicrystals of silicon have been deformed by symmetrical loading at temperatures which are moderate for covalent materials and correspond rather to cold deformation of most usual metals.

It has been shown that $\Sigma=9$ boundaries strongly hinder dislocation motion and hence slip propagation.

The possibility for dislocations to be absorbed in the grain boundary was confirmed by observations. But such absorption process does not lead to infinitesimal dislocations whose dispersion in the boundary plane would restore a perfect, although with different characteristics, interface. The absorption process is a reaction with the ordered structure of the grain boundary and leads to discrete grain boundary dislocations with Burgers vectors in the DSC lattice. The mobility of these DSC dislocations appears to be low except when it can occur by pure glide in the boundary plane. Such a low mobility prevents an effective reduction of pile ups and associated stress fields, through the conversion of crystal dislocations into grain boundary dislocations homogeneously distributed along the interface.

Dislocation transmission across the grain boundary was also evidenced thanks to specially designed experiments. This process appears to be very difficult since residual grain boundary dislocations have to be created, because of topological requirements, in the general case. This extra energy supply is possible only when large stress concentrations are present. Even for dislocations with a Burgers vector common to grains I and II and when a good matching of slip planes at the boundary exists, the dissociated nature of dislocations makes transmission at least as difficult as usual cross slip, since partial dislocations have not the same Burgers vectors in the two grains. Further, for dislocations whose Burgers vector must be changed before they can glide in the second crystal, transmission is rate controlled by the dispersion of residual dislocations in the boundary away from the impact and the process would saturate very rapidly if residual defects have a low mobility. As absorption, transmission cannot efficiently reduce pile up stresses.

TEM observations have proved that the pile up situation is rather resorbed by symmetric dislocation arrangements on either side of the boundary and by multiple slip (profuse cross slip, network formation with Lomer-Cottrell locks...). Various situations arise which however seem to obey general laws such as preservation of symmetry and minimization of 1 ong range internal stresses.

This is achieved by dislocation arrangements which are not unlike those observed at large strains in single crystals, except that they appear right at the onset of plastic deformation, at grain boundaries. The dislocation structure in the region adjacent to grain boundaries is a hardened (and hardening) one, compared with that which is observed in the grain interiors. In that sense one can say that near grain boundary regions are in an advanced stage of deformation.

There remains the question how representative our observations are for other crystal and grain boundary structures and for other deformation conditions.

Only grain boundary mechanisms, i.e. absorption and transmission, depend on the kind of grain boundary which is considered.

We feel, however, that dislocation transmission across grain boundaries is not important for the cold deformation of polycrystals, since this process was observed to be very difficult in the highly favourable situation of the $\Sigma=9$ boundary (one commor. Burgers vector and good matching of slip planes). Naturally, this conclusion should not apply for dislocation crossing of subgrain boundaries, which can be important under creep conditions.

Absorption of dislocations could be easier in grain boundaries with a smaller degree of coincidence than the "specia1" $\Sigma=9$. In "genera1" boundaries, dislocation Burgers vectors may be very small and the term "absorption" could mean that dislocations really lose most of their discreteness in the boundary. The absorption process would be increasingly important when temperature is increased. We feel that in cold deformation it is of 1 ittle importance, except may be to prevent crack formation at grain boundaries, through localized rearrangement of atoms at the head of a pile up for example.

The statement that hardened structures form at the grain boundary is believed to be valid in most cases dislocation arrangements do not depend on the grain boundary structure but rather on the geometrical restrictions that the crystallographic structure impo- 
ses to dislocation glide. One could suggest that the more glide is geometrically constrained within the bulk, the more the additionul geometric constraints, of the grain boundary will make themselves felt [27]. In example, one expects a greater difference between the dislocation structure near the grain boundary and that in the bulk for hcp crystals than for bcc crystals or for a fcc alloy with a low stacking fault energy than for $\mathrm{A} 1$ in which dislocations can glide in various types of planes. This, however, has still to be demonstrated.

Generally, stress fields at grain boundaries should be modified by elastic incompatibilities in anisotropic crystals and plastic incompatibilities resulting from non-symmetric loading. This would be taken care of by activating new slip systems which can prevail over those of highest Schmid factors near grain boundaries. In any case, this should be a further reason for hardened regions to form.

\section{Acknowledgements}

I am indebted to many colleagues from the Centre d'Etudes Nucléaires de Grenoble : J.J. Aubert, who grew the bicrystals, J.J. Bacmann, X. Baillin and J. Pelissier for HVEM experiments and many fruitful discussions, $\mathrm{N}_{i}$. El Kajbaji and J. Thibault-Desseaux for HREM observations and the permission to quote unpublished results. H.O.K. Kirchner and A. Korner, from the Institut für Festkörperphysik der Universität Wien, took a major part in TEM observations and the understanding of dislocation arrangements near grain boundaries. Thanks are also expressed to the staff of LURE-DCI for X-Ray experiments and to my coworkers from Nancy, Alain Jacques, Jean-Pierre Michel and Mario Martinez.

\section{References}

[1] Hirth, J.P., Metal1. Trans. A $\underline{3}$ (1972) 3047.

[2] Gleiter, H., Hornbogen, E., and Baro, G., Acta Metall. 16 (1968) 1053.

[3] Smith, D.A., J. de Physique 43 (1982) C6-225.

[4] Priester, L. in Les joints de grains dans les matériaux (1985) p.231, Les Editions de Physique.

[5] Lim, L.C., and Raj, R., J. de Physique 46 (1985, C4-581.

[6] Lartigue, S., and Priester, L., Acta Metall. 71 (1983) 1809.

[7] Hornstra, J., Physica 25 (1959) $409 ; 26$ (1960) 198.

[8] Krivanek, O.L., Isoda, S., and Kobayashi, K., Philos. Mag. A 36 (1977) 931.

[9] Mö1ler, H.J., Philos. Mag. A 43 (1981; 1045.

[10] D'Anterroches, C., and Bourret, A., Philos. Mag. A 49 (1984) 783 .

[11] Papon, A.M., Petit, M., and Bacmann, J.J., Philos. Mag. A 49 (1984) 573.

[12] Gratias, D., and Portier, R., J. de Physique 43 (1982) C6-15.

[13] Kalonji, G., J. de Physique 46 (1985) C4-249.

[14] King, A.H., and Smith, D.A., Acta Cryst. A $\underline{36}$ (1980) 335.

[15] King, A.H., Acta Meta11. 30 (1982) 419.

[16] El Kajbaji, M., Thesis (1986) Université de Grenoble.

[17] E1 Kajbaji, M., Thibault-Desseaux, J., MartinezHernandez, M., Jacques, A., and George, A.,
Rev. Phys. App1. (1987) to be published.

[18] Lim, L.C., Acfa Metali1. 35 (1987) 163.

[19] Pond, R.C., and Smith, D.A., Philos. Mag. A. 36 (1977) 353.

[20] Bacmann, J.J., Gay, M.o., and Tournemine, R., de Scripta Metall. 16 (1982) 353.

[21] Forwood, C.T., and Clarebrough, L.M., Philos. Mag. A 44 (1981) 31.

[22] Clark, W.A.T., and Wagoner, R.H., in "Dislocations in Solids" (1985) p.647, University of Tokyo Press.

[23] Hook, R.E., and Hirth, J.P., Acta Meta11. 15 (1967) 535.

[24] Hook, R.E., and Hirth, J.P., Acta Meta11. 15 (1967) 1099.

[25] Baillin, X., Peilissier, J., Bacmann, J.J., Jacques, A., and George, A., Philos. Mag. A 55 (1987) 143.

[26] Jacques, A., George, A., Baillin, X., and Bacmann, J.J., Philos. Mag. A $\underline{55}$ (1987) 165.

[27] Martinez-Hernandez, M., Kirchner, H.O.K., Korner, A., George, A., and Michel, J.P., Philos. Mag. A (1987) to be published.

[28] Wagoner, R.H., Metal1. Trans. A 12 (1981) 201:

[29] George, A., and Jacques, A., Proceedings of the Vth Symposium on structure and properties of dislocations in semiconductors, Moscow (1986) to be published.

[30] King, A.H., and Chen, F.R., Mater. Sci. Engng. 66 (1984) 227 .

[31] Prinz, F., Karnthaler, H.P., and Kirchner, H.0.K., Phys. Stat. So1.(a) 37 (1976) 223 .

[32] Hazzledine, P.M., Karnthaler, H.P., and Wintner, E., Philos. Mag. 32 (1975) 81. 